\title{
Modeling of Solidification Phenomenon in Casting-Mould System Including Shrinkage Cavities Formation
}

\author{
T. SkrzyPCZAK ${ }^{a, *}$ E. WęGrZYN-SkrzYPCZAK ${ }^{b}$ AND L. SoWA ${ }^{a}$ \\ ${ }^{a}$ Department of Mechanics and Fundamentals of Machine Design, \\ Czestochowa University of Technology, 42-201 Czestochowa, Poland \\ ${ }^{b}$ Department of Mathematics, Czestochowa University of Technology, \\ 42-201 Czestochowa, Poland \\ Doi: 10.12693/APhysPolA.138.308 \\ *e-mail: t.skrzypczak@imipkm.pcz.pl
}

\begin{abstract}
Mathematical model of solidification of cast steel solidification was presented in the paper. The description includes the phenomenon of heat transport from the casting to the mould through the air-filled gap of constant width as well as the cooling process of external walls of the mould. Governing equation of the model are the partial differential equations of transient heat diffusion in three-dimensional region supplemented by the additional boundary and initial conditions. Heat releasing during solidification has been taken into account by the modification of the specific heat of the solid-liquid mixture in the mushy zone. Finite element method (FEM) based on the standard Galerkin formulation was used to prepare the numerical model of considered phenomena. Two different finite element meshes were used for the casting and the mould, respectively. Internal macroscopic voids formation process due to volumetric shrinkage of solidifying material is an important part of the numerical model. These voids are called "shrinkage cavities" and are serious concern of the casting process. Theoretical basis was used to implement an original computer program which can be used to predict the quality of the castings. Computer simulation was carried out to confirm the application possibilities of the prepared model. The results of computer calculations of the solidification in three-dimensional casting-mould system were presented and discussed in details.
\end{abstract}

topics: heat transport, solidification, shrinkage cavity, numerical modeling, finite element method

\section{Introduction}

Volume loss of the alloy during solidification results in the micro- and macroscopic cavities scattered within the casting. Contraction of the material caused by the temperature decreasing is observed in the liquid and solid due to the temperature dependent density changes. Contraction before and after solidification has significantly less noticeable impact on the defect formation than the volume loss associated with the liquid-solid phase transition. This is the main reason of the formation process of defects observed in the micro- and macroscopic scales. Numerical models of shrinkage cavity formation process are widely discussed in the literature [1-8]. Most common macroscopic defects are conical pipe shrinkages observed in the top of the riser and also closed cavities located deeper in the casting $[1,2]$. Such voids are also called "shrinkage cavities". Presented paper is devoted to the numerical modeling of the solidification process taking into account the cooling impact of the mould and formation process of shrinkage cavities. Discussed model is useful in the prediction of the location and shape of multiple shrinkage cavities within single casting.
The total volume of the casting decreases during three steps of the process. First step begins after filling the mould with the liquid alloy and ends at the start of solidification. Pipe shrinkages formation can be affected by the contraction of the liquid caused by temperature decreasing. Presented work neglects mentioned step and only focuses on the stage of solidification. This process is observed between liquidus and solidus temperatures. Contraction of the casting during this step is mainly governed by the liquid-solid transformation. The liquid phase has a larger volume than solid because of its lower density. Liquid-solid transformation of the alloy leads to the shrinkage cavities formation in the casting. Depending on its shape and the cooling conditions the macroscopic gaseous cavities can be scattered inside the final product.

The contraction of the solid in the last stage of the casting process is caused by the temperature dependent increasing of the density and is also omitted in presented work.

\section{Mathematical and numerical models}

Figure 1 shows the casting-mould system divided into two parts $\Omega_{M}$ and $\Omega_{C}$, where $\Omega_{M}$ is the mould 


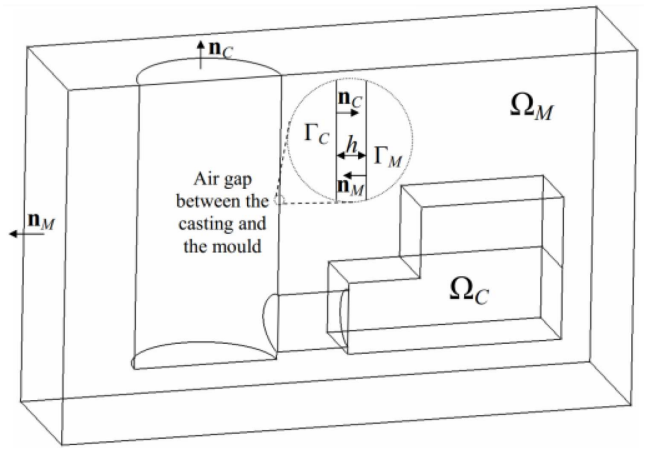

Fig. 1. Casting-mould system with the air gap.

region filled with cast iron, while $\Omega_{C}$ represents the casting filled with molten steel.

The external boundaries of the casting and the mould are represented by $\Gamma_{C}$ and $\Gamma_{M}$, respectively. The part $\Omega_{C}$ contains solidifying alloy from where the heat is transported to the mould and also to the ambient. Between the casting and the mould one can observe the air gap filled with gaseous medium. In general case the width of the gap varies according to the thermal deformations of the casting and the mould but in presented model constant width of the gap is assumed. Mathematical model is based on the differential equations of heat transport in the casting and the mould, namely

$$
\begin{gathered}
\frac{\partial}{\partial x}\left(\lambda_{C} \frac{\partial T^{C}}{\partial x}\right)+\frac{\partial}{\partial y}\left(\lambda_{C} \frac{\partial T^{C}}{\partial y}\right) \\
+\frac{\partial}{\partial z}\left(\lambda_{C} \frac{\partial T^{C}}{\partial z}\right)=c^{*} \rho_{C} \frac{\partial T^{C}}{\partial t} \\
\frac{\partial}{\partial x}\left(\lambda_{M} \frac{\partial T^{M}}{\partial x}\right)+\frac{\partial}{\partial y}\left(\lambda_{M} \frac{\partial T^{M}}{\partial y}\right) \\
+\frac{\partial}{\partial z}\left(\lambda_{M} \frac{\partial T^{M}}{\partial z}\right)=c_{M} \rho_{M} \frac{\partial T^{M}}{\partial t}
\end{gathered}
$$

where $T[\mathrm{~K}] \quad$ represents temperature,

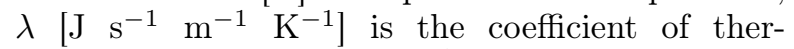
mal conduction, $\rho\left[\mathrm{kg} / \mathrm{m}^{3}\right]$ represents density, $c *\left[\mathrm{~J} \mathrm{~kg}^{-1} \mathrm{~K}^{-1}\right]$ is effective specific heat, $L\left[\mathrm{~J} \mathrm{~kg}^{-1}\right]$ is latent heat of solidification, $x, y, z[\mathrm{~m}]$ are spatial coordinates, $t[\mathrm{~s}]$ is represents time, while $C$ and $M$ are related to the casting and the mould.

According to [9] parameter $c_{j}^{*}$ can be defined in the following way:

$$
c_{j}^{*}= \begin{cases}c_{S}, & T<T_{S} \\ \frac{c_{S}+c_{L}}{2}+\frac{L}{T_{L}-T_{S}}, & T_{S} \leq T \leq T_{L} \\ c_{L}, & T>T_{L}\end{cases}
$$

where $c_{L}\left(c_{S}\right)$ is the specific heat of the liquid (solid), $T_{L}\left(T_{S}\right)$ represents the liquidus (solidus) temperature, and $L[\mathrm{~J} / \mathrm{kg}]$ is the latent heat of solidification.

Above mathematical model must be complemented by the boundary and initial conditions:

$$
T^{C}(t=0)=T_{0}^{C}, \quad T^{M}(t=0)=T_{0}^{M},
$$

$$
\begin{aligned}
& -\lambda_{C} \frac{\partial T^{C}}{\partial n_{C}}=\alpha_{C}\left(T^{C}-T_{\infty}^{C}\right), \\
& -\lambda_{M} \frac{\partial T^{M}}{\partial n_{M}}=\alpha_{M}\left(T^{M}-T_{\infty}^{M}\right), \\
& -\lambda_{M} \frac{\partial T^{M}}{\partial n_{M}}=\frac{\lambda_{g}}{h}\left(T^{M}-T^{C}\right)=\lambda_{C} \frac{\partial T^{C}}{\partial n_{C}},
\end{aligned}
$$

where $T_{\infty}[\mathrm{K}]$ is the ambient temperature, $\alpha\left[\mathrm{J} /\left(\mathrm{s} \mathrm{m}^{2} \mathrm{~K}\right)\right]$ represents the coefficient of heat transfer, $n$ is the direction of the vector perpendicular to the boundary, $\lambda_{g}$ represents the coefficient of thermal conduction of the medium in the gap, $h[\mathrm{~m}]$ denotes width of the gap.

Each equation in (1) is discretized with respect to the spatial coordinates with the use of standard Galerkin formulation. Then, the Euler's backward time integration scheme is used to derive global set of linear equations:

$$
\begin{gathered}
\left(\boldsymbol{K}_{T M}+\frac{\boldsymbol{M}_{T M}}{\Delta t}\right) \boldsymbol{T}_{M}^{f+1}=\boldsymbol{B}_{T M}+\frac{\boldsymbol{M}_{T M}}{\Delta t} \boldsymbol{T}_{M}^{f} \\
\left(\boldsymbol{K}_{T C}+\frac{\boldsymbol{M}_{T C}}{\Delta t}\right) \boldsymbol{T}_{C}^{f+1}=\boldsymbol{B}_{T C}+\frac{\boldsymbol{M}_{T C}}{\Delta t} \boldsymbol{T}_{C}^{f}
\end{gathered}
$$

where $\boldsymbol{K}_{T C}, \boldsymbol{K}_{T M}$ are the global matrices of thermal conductivity, $\boldsymbol{M}_{T C}, \boldsymbol{M}_{T M}$ represent global matrices of thermal capacity, $\boldsymbol{B}_{T C}, \boldsymbol{B}_{T M}$ are the vectors containing boundary conditions, $f$ denotes the number of time step $\Delta t$.

The process of shrinkage cavities formation is gravity dependent. It starts when the first portion of the solid phase appearing in the casting. Numerically the formation of shrinkage cavities is based on the nodes of finite element mesh. Each node is initially filled with particular amount of liquid material. Total sum of the nodal volumes is the same as the volume of entire casting. During the time step the following procedures must be done in order to grow the shrinkage cavities:

- The contraction of the material is calculated as the volume of the solid phase $\Delta V_{S}\left[\mathrm{~m}^{-3}\right]$ appearing in the current time step multiplied by the contraction coefficient $S_{h}$. The value of $\Delta V_{S}$ is calculated separately for the each closed liquid zone in the casting.

- Starting from the highest node in the each liquid zone (nodes are sorted with respect to vertical coordinate) the liquid phase is replaced by the air.

- If the calculated volume of the air is less than nodal volume the previous procedure is skipped and calculation process goes to the next step.

The calculation process is finished when the last amount of the liquid turns into the solid. The algorithm makes possible to simulate growing process of multiple cavities within the casting. The number of cavities depends on the number of closed liquid zones forming inside the casting during 
solidification process. In the each time step the temporary distribution of temperature in the mould and the casting is obtained independently with the use of separated finite element meshes.

\section{Example of calculations}

The original computer program based on the FEM is used to simulate described process. Temporary temperature distribution and formation process of the shrinkage cavities are investigated. The thermal material properties of the mould and the air in the gap are the same as in the paper [10] while properties of the casting are taken from [11].

The mould of dimensions $0.4 \times 0.3 \times 0.2 \mathrm{~m}^{3}$ is initially filled with melted alloy of temperature $T_{0}=1800 \mathrm{~K}$. Initial temperature of the mould equals $600 \mathrm{~K}$. Because only half of the mould is taken into account thermal insulation is assumed on the plane of symmetry while the coefficient of heat transfer is defined on the external boundaries $\alpha=20 \mathrm{~J} /\left(\mathrm{s} \mathrm{m}^{2} \mathrm{~K}\right)$ together with the ambient temperature $T_{\infty}=300 \mathrm{~K}$. Constant time step $\Delta t$ equals $0.25 \mathrm{~s}$. The meshes for the casting and the mould are built of tetrahedrons and contain 47968 and 58397 nodes, respectively.

Figures 2-4 show temporary temperature distribution in the casting and the mould and also the macroscopic defect formation (marked as green). During the early stage of solidification first shrinkage cavity appears in the upper left part of the casting. When the narrow connection between left and right parts of the casting is filled with the mixture of the liquid and solid phases the liquid cannot flow to the right part and the second defect starts to grow (Fig. 3). Figure 4 shows temperature distribution at the end of solidification. Fully developed shrinkage cavities are visible. Defect on the left is opened while the second one is closed and located deeper in the casting. Total time of solidification equals about $180 \mathrm{~s}$ due to rapid heat transport from the casting to the permanent mould.

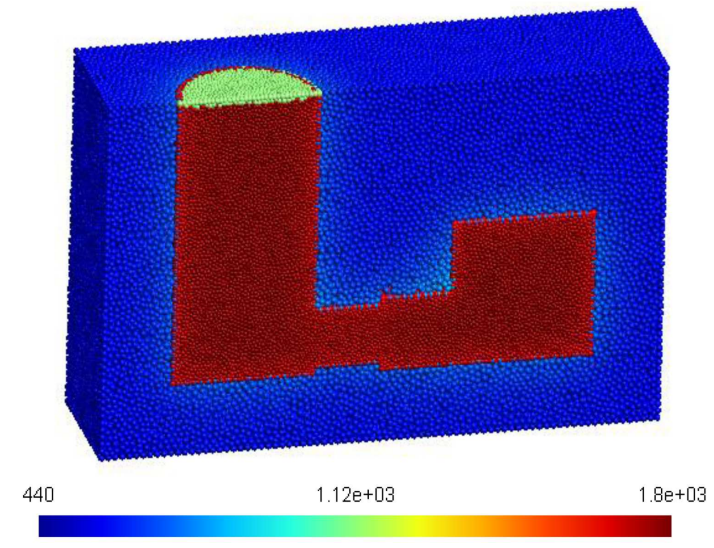

Fig. 2. Temperature distribution and shrinkage cavity after $25 \mathrm{~s}$

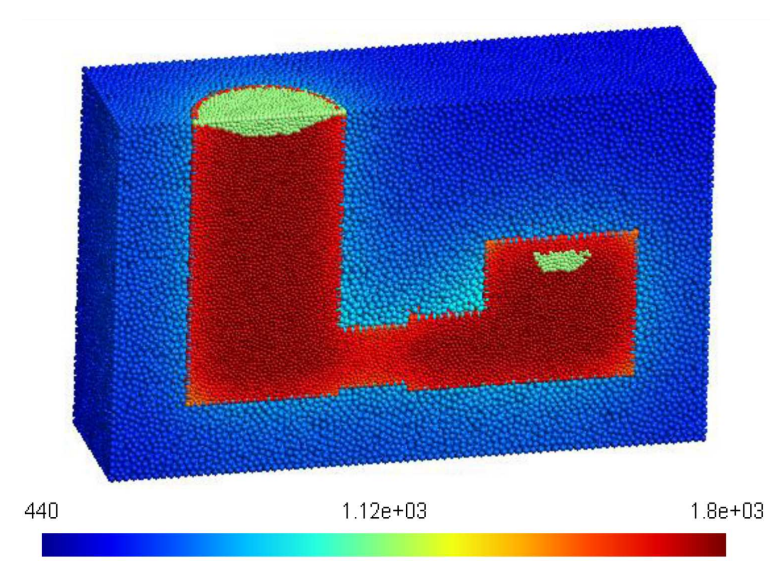

Fig. 3. Temperature distribution and shrinkage cavity after $125 \mathrm{~s}$.

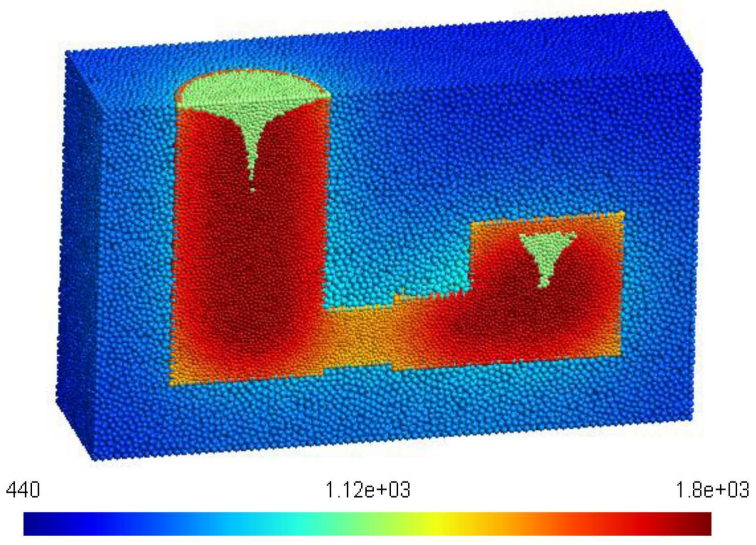

Fig. 4. Temperature distribution and shrinkage cavity after $200 \mathrm{~s}$.

\section{Conclusions}

The basis of the mathematical and numerical models of solidification process in the casting-mould system with the shrinkage cavity formation process was presented. The results of computer simulation show the effectiveness of the solver. Presented model is useful for the simulations of multiple macroscopic cavities. Original computer program is effective for complex three-dimensional castings. It is also more flexible than widely used commercial packages. Further work in this field is devoted to the experimental validation of the obtained results.

\section{References}

[1] J. Hajkowski, P. Roquet, M. Khamashta, E. Codina, Z. Ignaszak, Arch. Foundry Eng. 17, 57 (2017).

[2] Ch. Pequet, M. Rappaz, M. Gremaud, Metall. Mater. Trans. A 33, 2095 (2002).

[3] M.C. Flemings, The solidification processing, Mc Graw-Hill, New York 1974. 
[4] J. Campbell, Castings, 2nd ed., Butterworth-Heinemann, Oxford 2003.

[5] M. Wu, A. Ludwig, A. Kharicha, Appl. Math. Model. 41, 102 (2017).

[6] C. Zhang, Y. Bao, M. Wang, L. Zhang, Arch. Foundry Eng. 16, 27 (2016).

[7] C.J. Kim, S.T. Ro, J. Heat. Trans. 115, 1078 (1993).

[8] A.S. Jabur, F.M. Kushnaw, J. Appl. Computat. Math. 6, 7 (2017).
[9] B. Mochnacki, J. S. Suchy, Numerical Methods in Computations of Foundry Processes, Polish Foundrymen's Technical Association, Kraków 1993.

[10] T. Skrzypczak, E. Wegrzyn-Skrzypczak, L. Sowa, Arch. Foundry Eng. 17, 147 (2017).

[11] T. Skrzypczak, L. Sowa, E. WęgrzynSkrzypczak, Arch. Foundry Eng. 20, 37 (2020). 